\title{
Charmonium resonances on the lattice
}

\author{
Gunnar Bali ${ }^{1}$, Sara Collins ${ }^{1}$, Daniel Mohler ${ }^{4,5}$, M. Padmanath ${ }^{1}$, Stefano Piemonte ${ }^{1, \star}$, Sasa \\ Prelovsek $^{1,2,3}$, and Simon Weishäupl ${ }^{1, \star \star}$ \\ ${ }^{1}$ Institute for Theoretical Physics, University of Regensburg, 93040 Regensburg, Germany \\ ${ }^{2}$ Faculty of Mathematics and Physics, University of Ljubljana, 1000 Ljubljana, Slovenia \\ ${ }^{3}$ Jozef Stefan Institute, 1000 Ljubljana, Slovenia \\ ${ }^{4}$ Helmholtz-Institut Mainz, 55099 Mainz, Germany \\ ${ }^{5}$ Johannes Gutenberg Universität Mainz, 55099 Mainz, Germany
}

\begin{abstract}
The nature of resonances and excited states near decay thresholds is encoded in scattering amplitudes, which can be extracted from single-particle and multiparticle correlators in finite volumes. Lattice calculations have only recently reached the precision required for a reliable study of such correlators. The distillation method represents a significant improvement insofar as it simplifies quark contractions and allows one to easily extend the operator basis used to construct interpolators. We present preliminary results on charmonium bound states and resonances on the $N_{f}=2+1$ CLS ensembles. The long term goal of our investigation is to understand the properties of the $\mathrm{X}$ resonances that do not fit into conventional models of quark-antiquark mesons. We tune various parameters of the distillation method and the charm quark mass. As a first result, we present the masses of the ground and excited states in the $0^{++}$and $1^{--}$channels.
\end{abstract}

\section{Introduction}

The mysterious properties of exotic QCD resonances have motivated an increasing interest in nonperturbative lattice calculations during the last decade. Monte-Carlo simulations of QCD regularized on the lattice can provide an alternative insight into the fundamental mechanisms of strong interactions, but the study of resonances is a challenge that requires a huge numerical and theoretical effort. In particular, scattering of particles cannot be investigated directly on an Euclidean space-time lattice, but, following the Lüscher method [1], scattering amplitudes are instead extracted from the finite volume dependence of the discrete energy levels of the spectrum of two interacting particles in a box. Many simulations with either different volumes or in different moving frames are needed and a large number of configurations is required to measure the lattice energy levels with a sufficient precision to compute scattering observables. Only recently lattice QCD calculations have been able to reach sufficiently small lattice spacings and pion masses to allow for a reliable study of QCD resonances with heavy flavor content.

The aim of our project is the understanding of the nature of charmonium(-like) resonances and excited states near decay thresholds by means of non-perturbative lattice simulations. In this contribution we present our recent progress, regarding the calculation of the energy levels on the $N_{f}=2+1$

\footnotetext{
${ }^{\star}$ Speaker, e-mail: stefano.piemonte@physik.uni-regensburg.de

${ }^{\star}$ Speaker, e-mail: simon.weishaeupl@physik.uni-regensburg.de
} 
ensembles generated by the CLS collaboration. We employ the distillation method, which significantly improves the computation of all diagrams involving single-meson and two-meson correlation functions. In Section 2 we present the current experimental status, the results of previous lattice investigations and the motivations for our project. In Section 3 we describe the details of our ensembles and the tuning of the charm quark mass, while in Section 4 we give an overview of the distillation method and the parameters used in our analysis. Our results are presented in Section 5 and are followed by the conclusions in Section 6.

\section{Experimental status and previous lattice investigations}

Our final goal is to consider conventional and exotic charmonium(-like) resonances. Here we focus on the vector and scalar channels. The resonances are labeled "XY" states, the letter $Y(\ldots)$ is used to denote the states with quantum numbers $1^{--}$, while the letter $X(\ldots)$ is used for all other flavorless charmonium exotic resonances.

The two lightest states of the charmonium spectrum in the vector channel, the $J / \psi$ and $\psi(2 S)$, are relatively stable and decay by charm-quark annihilation or weak interactions. The third state, the $\psi(3770)$, is a resonance which decays by strong interactions, lying above the $\bar{D} D$ decay threshold. The decay modes of the $\psi(3770)$ are in agreement with those of an excitation of a quark-antiquark meson. At higher energies, there are many other states whose structure and constituents are unknown, like the $Y(4260)$, where BESIII recently found two peaks $Y(4240)$ and $Y(4320)$ [2]. The higher peak is consistent with $Y(4360)$ reported earlier. Our first step toward the study of these exotic states is to investigate the resonance $\psi(3770)$, whose properties are well-known experimentally and that can be used as a benchmark to estimate carefully the systematic uncertainties of our computation of the phase shift.

The $\chi_{c 0}$ is the lightest bound state in the charmonium spectrum with quantum numbers $0^{++}$. The higher lying $X(3915)$ was discovered by Belle in 2004 in $J / \psi \omega$ decays [3] and was subsequently listed as the first excited state $\chi_{c 0}(2 P)$ by the Particle Data Group [4]. This identification was based on a determination of its quantum numbers by BaBar [5], and has since been challenged. The OZI rule favors an excited $\bar{c} c$ state decaying into $D \bar{D}$ mesons, but not via $J / \psi \omega[6]$ and, at present, there is still no evidence for a resonance at $3915 \mathrm{MeV}$ in the s-wave $D \bar{D}$ meson scattering channel. Considering "exotic" interpretations of the state, a $\bar{c} c$-gluon exotic hybrid scenario has been excluded by lattice calculations as such states appear to be significantly heavier than all other low energy resonances [7]. The suppression of $D \bar{D}$ meson decays could instead be explained easily if the $X(3915)$ has a hidden strange content. A possible structure could be in this case a bound state of two $D_{s}$ mesons, and the $20 \mathrm{MeV}$ gap of $X(3915)$ from the $\bar{D}_{s} D_{s}$ decay threshold would be interpreted as the binding energy of the meson molecule. The decays into $J / \psi \omega$ could be explained in terms of the $\omega-\phi$ mixing [8]. Even in this case it is still difficult to explain why the $X(3915)$ has not yet been seen in the $\eta \eta_{c}$ decay channel, given the larger mixing in pseudoscalar light-strange sector with respect to the vector case [9]. An appealing hypothesis is that the $X(3915)$ is a $c s \bar{c} \bar{s}$ tetraquark, i.e. a bound state with four valence quarks without a definite internal structure.

Another solution of this puzzle has been suggested in [10], where the authors argue that BaBar made an unjustified assumption in their analysis, which results in the exclusion of $J^{P C}=2^{++}$as the quantum numbers for the $X(3915)$. Indeed the authors argue that the $X(3915)$ is simply the already known $\chi_{c 2}(2 P)$ [11]. If the $X(3915)$ is not the first excited state of the $\chi_{c 0}$, there is the question of what the energy of such a state could be. The Belle collaboration has recently reported a new scalar charmoniumlike state $X^{*}(3860)$ that decays into $D \bar{D}$ mesons as expected for the excited state of the $\chi_{c 0}[12]$. 


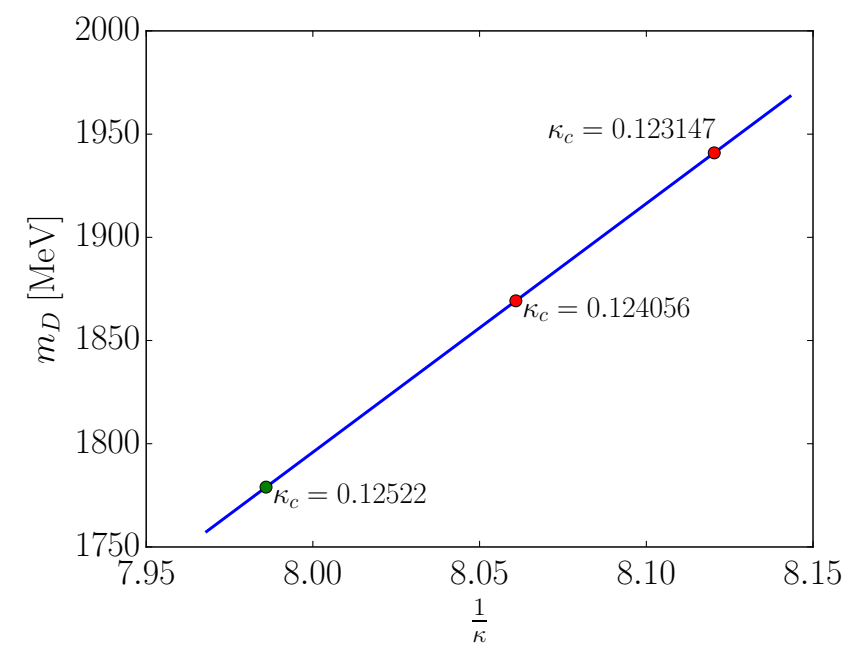

Figure 1. Dependency of the D-meson mass $m_{D}$ on the bare charm quark mass $m$ for different $\kappa_{c}$ - values. The two red points indicate the previously tuned values of $\kappa_{c}$. The blue line indicates a linear fit to determine the new value $\kappa_{c}=0.125220$ corresponding to a D-meson with a mass $80 \mathrm{MeV}$ lighter than its physical value.

The lack of a deep knowledge of the scalar and vector exotic charmonium resonances is a strong motivation for non-perturbative lattice calculations of the scattering matrix. Previous investigations performed by some of us have been able to extract the elastic scattering phase shift from a basis including both quark-antiquark and $D \bar{D}$ interpolators in the scalar channel [13]. However, the study of the resonance structure did not resolve the issue of the first excited scalar charmonium. These exploratory results, which did not consider strange quarks in the valence or the sea sector, gave an indication for a bound state $\chi_{c 0}$ and a rather narrow resonance slightly below $4 \mathrm{GeV}$. More simulations are required to reach a conclusive picture of the scalar resonances, particularly to address the experimental $X^{*}(3860)$ and $X(3915)$ states. We plan to extend the previous analysis by considering also a coupling to a hidden strange sector. This will entail coupled scattering of $\bar{D} D$ and $\bar{D}_{s} D_{s}$ channels.

\section{Lattice Setup}

We perform our analysis on the $N_{f}=2+1$ ensembles generated by the CLS collaboration, see, for example, Refs. [14] and [15] for details. Two ensembles have been so far considered, labeled as U101 and $\mathrm{H} 105$, both with $m_{\pi}=280 \mathrm{MeV}$ and lattice spacing $a=0.0854 \mathrm{fm}$, but with different lattice volumes of $24^{3} \times 128$ and $32^{3} \times 96$, respectively. Our lattice discretization of the QCD action includes non-perturbatively $O(a)$-improved Wilson fermions with tree-level Symanzik improved gauge action. Open boundary conditions have been applied to the fermion and gluon fields in the time direction, while they are periodic in space.

The strange and the light quarks are dynamical and their masses are fixed in the Monte-Carlo simulation, however, we have the freedom to choose the mass of the (quenched) charm quark. The hopping parameter $k_{c}$, where the bare quark mass $a m_{c}=\left(1-8 k_{c}\right) /\left(2 k_{c}\right)$, is usually fixed so that the experimental value of the spin average of the $J / \psi$ and the $\eta_{c}$ meson masses is reproduced. For the current project, for which we employ ensembles with unphysical pion masses, this may result in the $\psi(3770)$ lying below the $D \bar{D}$ threshold, so that the s-wave decay into $D \bar{D}$ mesons would be forbidden. 


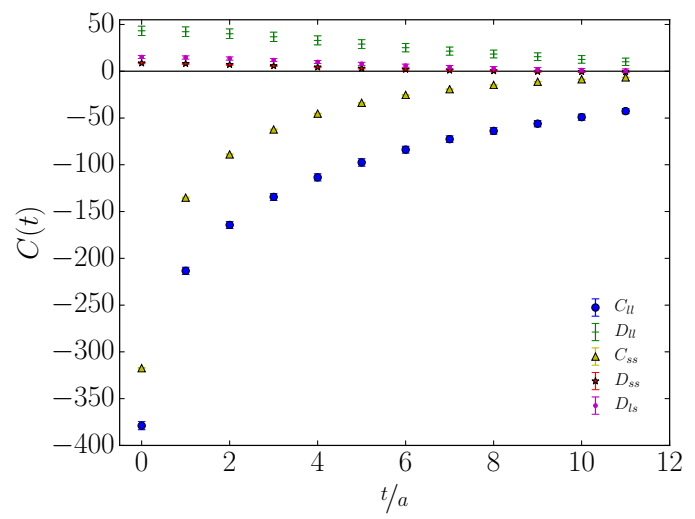

(a) Connected and disconnected pseudoscalar correlators

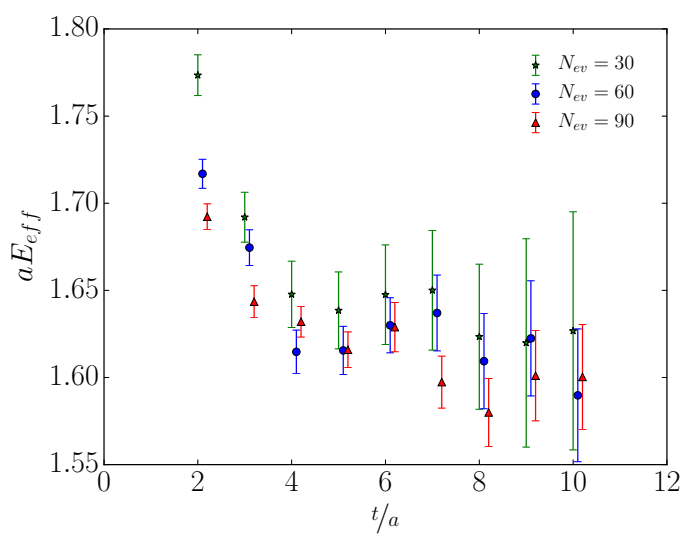

(b) Effective mass of the first excited state of the $\eta_{c}$

Figure 2. 2(a) Trace of the contribution to the pseudoscalar correlator for strange and light quarks (related to $\eta$ and $\eta^{\prime}$ mesons). The disconnected contributions $D_{f f}$ for the dynamical flavors $f$ are smaller in magnitude but have a larger statistical uncertainty than the connected contributions $C_{f f}$. 2(b) Effective mass plots for the first excited charmonium state $\left(0^{-+}\right)$using 30,60 and 90 Laplacian eigenvectors.

This scenario might be avoided by reducing the charm quark mass. We therefore use two different $k_{c}$ interpolating between a $D$ meson mass approximatively $80 \mathrm{MeV}$ above and below its physical value to try estimate how the energy levels move with respect to the threshold as a function of the charm quark mass, see Fig. 1.

\section{Distillation method and variational analysis}

To study the charmonium spectrum, we use the distillation method (quark-field smearing) [16] and the variational analysis of the correlation matrix between single and multi-particle operators at different timeslices. The wave function is constructed from the eigenvectors of the $3 \mathrm{D}$ Laplace operator $\nabla_{t}^{2}$ defined on a given timeslice $t$. A fermion wave function defined on a single lattice point can be smeared by the "distillation" operator

$$
\square(t)=\sum_{i=0}^{N} \Psi_{i}(t) \Psi_{i}^{\dagger}(t),
$$

where the sum runs over the eigenvectors $\Psi_{i}(t)$ of $\nabla_{t}^{2}$ corresponding to the $N$ lowest eigenvalues. The resulting wave function has a Gaußian bell shape, whose width is controlled by the number of eigenvectors included in the sum, typically of the order of $O(100)$. If $N$ is taken as large as $3 \times V_{3}$, to span the entire linear space, then the distillation operator reduces to the identity operator and no smearing is applied at all.

In the distillation method, a generic two-point correlation function can be rewritten as a product of a combination of at least four matrices

$$
C\left(t, t_{0}\right)=\operatorname{Tr}\left[\phi^{B}(t) \tau\left(t, t_{0}\right) \phi^{A}\left(t_{0}\right) \tau\left(t_{0}, t\right)\right] .
$$


Table 1. Interpolating operators for the $1^{--}$(left) and the $0^{++}$(right) channel. All repeated indexes are summed over the spatial components and derivatives are implemented in a symmetric form.

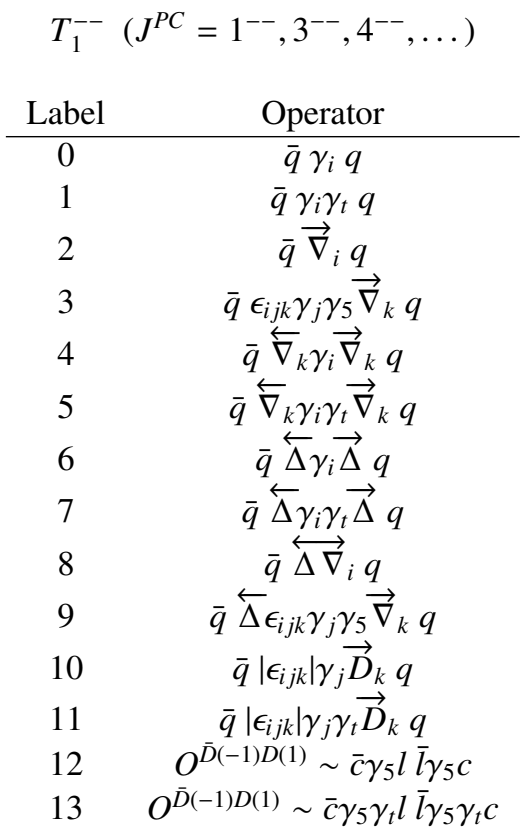

$$
A_{1}^{++}\left(J^{P C}=0^{++}, 4^{++}, \ldots\right)
$$

\begin{tabular}{cc} 
Label n & Operator \\
\hline 0 & $\bar{q} q$ \\
1 & $\bar{q} \gamma_{i} \vec{\nabla}_{i} q$ \\
2 & $\bar{q} \gamma_{i} \gamma_{t} \vec{\nabla}_{i} q$ \\
3 & $\bar{q} \overleftarrow{\nabla}_{i} \vec{\nabla}_{i} q$ \\
4 & $\bar{q} \overleftarrow{\Delta \Delta} q$ \\
5 & $\bar{q} \overleftarrow{\Delta} \gamma_{i} \vec{\nabla}_{i} q$ \\
6 & $\bar{q} \overleftarrow{\Delta} \gamma_{i} \gamma_{t} \vec{\nabla}_{i} q$ \\
7 & $O^{\bar{D}(0) D(0)} \sim \bar{c} \gamma_{5} l \bar{l} \gamma_{5} c$ \\
8 & $O^{\bar{D}(0) D(0)} \sim \bar{c} \gamma_{5} \gamma_{t} l \bar{l} \gamma_{5} \gamma_{t} c$ \\
9 & $O^{\bar{D}(p) D(-p)} \sim \bar{c} \gamma_{5} l \bar{l} \gamma_{5} c$ \\
10 & $O^{\bar{D}^{*}(0) D^{*}(0)} \sim \bar{c} \gamma_{i} l \bar{l} \gamma_{i} c$ \\
11 & $O^{\bar{D}^{*}(0) D^{*}(0)} \sim \bar{c} \gamma_{i} \gamma_{t} l \bar{l} \gamma_{i} \gamma_{t} c$ \\
12 & $O^{J / \psi(0) \omega(0)} \sim \bar{c} \gamma_{i} c \bar{l} \gamma_{i} l$ \\
13 & $O^{J / \psi(0) \omega(0)} \sim \bar{c} \gamma_{i} \gamma_{t} c \bar{l} \gamma_{i} \gamma_{t} l$ \\
14 & $O^{\bar{D}_{s}(0) D_{s}(0)} \sim \bar{c} \gamma_{5} s \bar{s} \gamma_{5} c$ \\
15 & $O^{\bar{D}_{s}(0) D_{s}(0)} \sim \bar{c} \gamma_{5} \gamma_{t} s \bar{s} \gamma_{5} \gamma_{t} c$
\end{tabular}

The perambulators $\tau$, describing the fermion propagation between eigenvectors at possibly different timeslices

$$
\tau\left(t, t_{0}, i, j\right)_{\alpha \beta}=\Psi_{i}^{\dagger}(t) D_{\alpha \beta}^{-1} \Psi_{j}\left(t_{0}\right),
$$

are precomputed for the charm, the light and the strange quarks. The total number of inversions required is $4 \times N$. Afterwards, the perambulators are combined with the $\phi^{\prime} s$

$$
\phi^{A}(t, i, j)_{\alpha \beta}=\Psi_{i}^{\dagger}(t) \Gamma^{A}(t)_{\alpha \beta} \Psi_{j}(t),
$$

that carry all information about source/sink operators and quantum numbers. Finally, the matrices $\phi$ and $\tau$ are multiplied and traced accordingly to Eq. 2 to construct single-meson correlators and the more involved expressions are required for multi-meson correlators. We always include diagrams with disconnected or backtracking strange and light loops, neglecting such contributions only for the charm. The disconnected contributions for the light quarks are a typical source of large statistical fluctuations in the correlation functions, see Fig. 2(a).

The first step of the distillation method is to choose the number of eigenvectors $N$. The optimal width of the wave function for charmonium states is expected to be smaller than that of light mesons. One therefore needs a larger number of eigenvectors compared to similar analyses of light resonances. Our strategy for searching for the optimal value of $N$ is to look for the point where an increase of $N$ does not result to a reduction in the error of the first excited state of the $\eta_{c}$, see Fig. 2(b). The number of eigenvectors $N$ has been set to 90 for the U101 ensemble and to 150 for the H105.

Once the Laplacian eigenvectors are available, operators and perambulators can be computed in parallel. So far we have implemented rest frame operators, see the list in Tab. 1. Operators relevant 


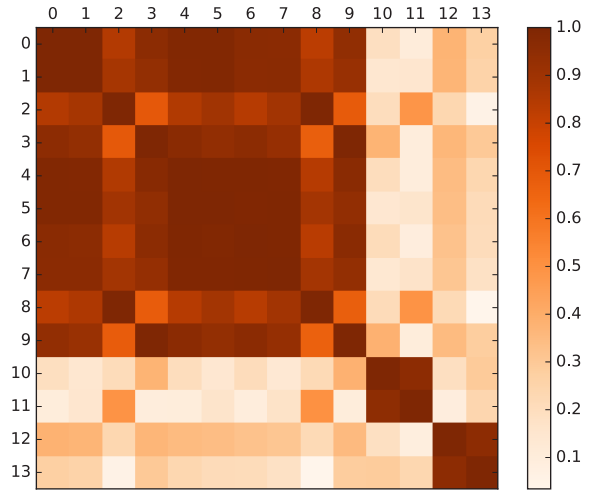

(a) Vector channel

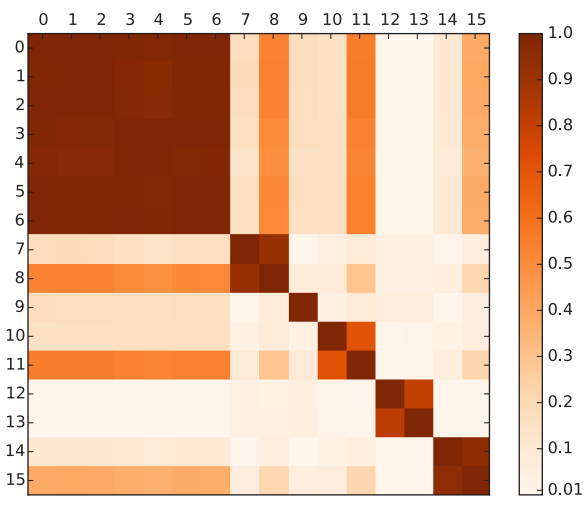

(b) Scalar channel

Figure 3. Matrix plots of the normalized correlation matrix $M_{i j}(t)$ in Eq. (8) at timeslice $t=5 a$ for all operators in the $1^{--}$(left) and the $0^{++}$(right) channels from table 1 .

for moving frames are being developed [17] and will be employed in the near future. The energy levels are finally computed from the solution of the generalized eigenvalue problem of the correlation matrix

$$
C_{i j}(t)=\left\langle O_{i}(t) O_{j}^{\dagger}(0)\right\rangle,
$$

as the result of the fit to an exponential decay of the eigenvalues

$$
\lambda_{m}\left(t, t_{0}\right) \propto e^{-\left(t-t_{0}\right) E_{m}}\left(1+O\left(e^{-\left(t-t_{0}\right) \Delta E_{m}}\right)\right)
$$

from

$$
C(t) v_{m}\left(t, t_{0}\right)=\lambda_{m}\left(t, t_{0}\right) C\left(t_{0}\right) v_{m}\left(t, t_{0}\right) .
$$

We fix $t_{0}=2$ and we optimize the choice of the basis of operators by looking at the normalized correlation matrix

$$
M_{i j}(t)=\frac{C_{i j}(t)}{\sqrt{C_{i i}(t) C_{j j}(t)}} .
$$

The structure of the matrix $M$ can help identify how the operators are correlated and how different states can be effectively separated from each others by a suitable choice of the operator basis. From Fig. 3, in the vector channel we do not see strong correlations between two- and single-particle operators, while a signal for stronger correlations appears in the scalar channel.

Translational invariance in the time direction is broken on gauge-field configurations with open boundary conditions. Correlators must be computed with source positions at least 28 timeslices away from the boundaries, a value chosen by looking for boundary effects in the pion correlator. In addition, we average on each configuration the correlators for eight different timesources, well separated in order to reduce the autocorrelation. Finally, two consecutive analyzed configurations are separated by twenty molecular dynamics units and ten accept/reject Metropolis steps. 


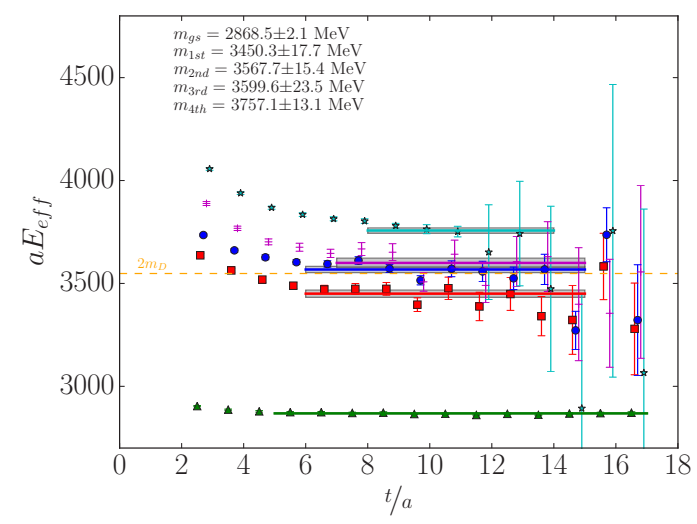

(a) $\kappa_{c}=0.125220$

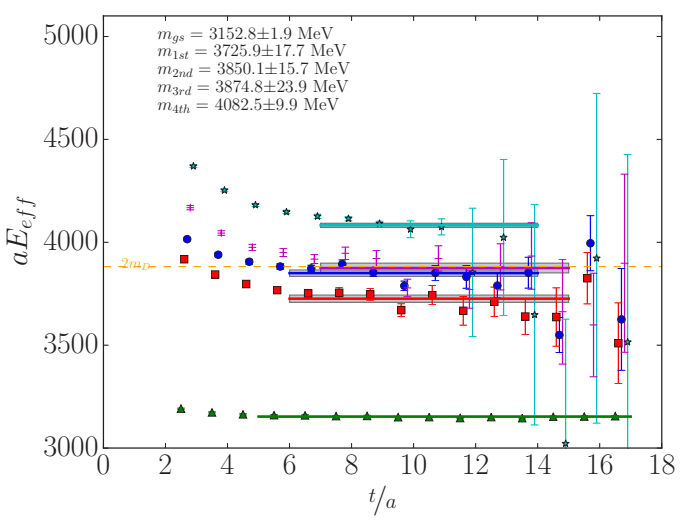

(b) $\kappa_{c}=0.123147$

Figure 4. Effective masses of the 5 lowest states in the $1^{--}$channel with $\kappa_{c}=0.125220$ and $\kappa_{c}=0.123147$, extracted with the variational analysis method from a basis of 10 operators $\{0,1,4,5,7,8,10,11,12,13\}$. The results are obtained from 125 configurations of the U101 ensemble.

\section{Results}

The analysis of the two ensembles is not yet completed, therefore at the moment we can present only the effective energy levels of the ensemble U101 for total momentum zero. Our plans for the near future is to double the statistics on the U101 ensembles and to perform all contractions including operators in moving frames; (full) distillation is used in this case. An analogous study is planned on ensemble H105 with larger volume, where the perambulators are already computed with stochastic distillation [18]. The final analysis will also have to consider the impact of the reweighting factors of the positive twisted mass introduced to stabilize the HMC trajectory, which have not yet been included. Finally, the finite volume energies will be computed, and the scattering amplitudes will be suitably parameterized. The main challenge will be the understanding of how the various decay channels are coupled together for the $0^{++}$resonances.

Nevertheless, we can already discuss the structure of the discrete spectrum of energy levels and the impact of tuning the charm quark mass. In the $1^{--}$channel, we are able to reliably extract five energy levels in total, see Fig. 4. The entire charmonium spectrum shifts as expected if the charm quark mass is decreased. The comparison of the two different $\kappa_{c}$ reveals that $E_{3}-2 m_{D}$ is bigger on $k_{c}=0.125220$ than on $k_{c}=0.123147$, where $E_{3}$ is related to $\psi(3770)$. It is therefore more likely that $\psi(3770)$ is a resonance above threshold in the former case. The statistical significance is still not high enough to draw a complete conclusion on this point, and the determination of the error is still preliminary. It is however clear that the systematic uncertainty of the tuning of the $\kappa_{c}$ does not dramatically affect the physical picture that we aim to investigate, but that a clever choice of the charm quark mass can help to move the $\psi(3770)$ toward a resonance state.

The energy levels in the $0^{++}$channel are presented in Fig. 5. As expected, the inclusion of hidden strange sector operators in our basis results in an additional scattering energy level above the one given by $\bar{D} D$ in s-wave at zero momentum. At the physical point, the two thresholds of $\bar{D}_{s} D_{s}$ and $\bar{D} D$ meson scattering are well separated by approximatively $200 \mathrm{MeV}$. In our setup, extrapolating along the constant $2 m_{l}+m_{s}$ line, the gap between the two corresponding scattering levels is reduced to about $100 \mathrm{MeV}$. This effect is driven by the fact that we are working with a heavier than physical 


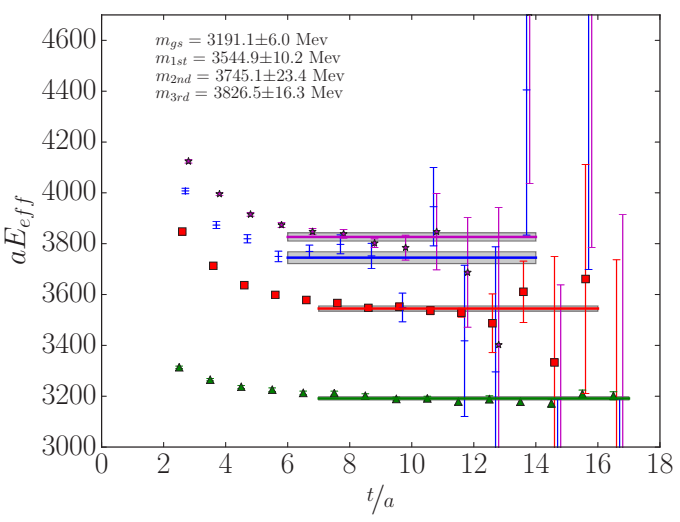

(a) $\kappa_{c}=0.125220$ excluding strange

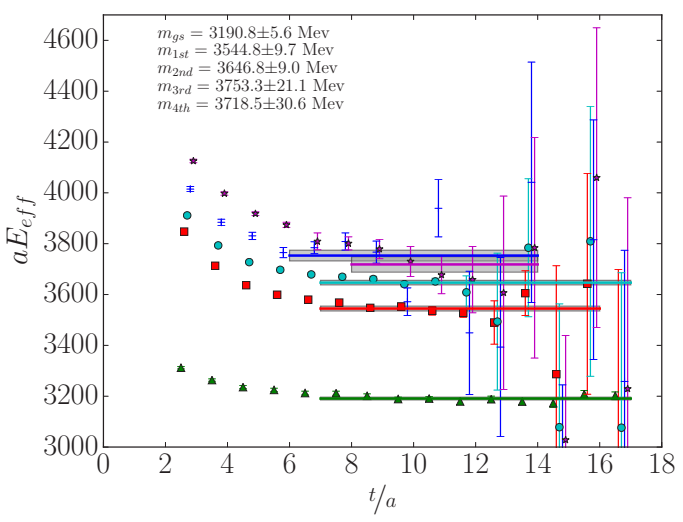

(b) $\kappa_{c}=0.125220$ including strange

Figure 5. Effective masses of the 4 lowest states in the $0^{++}$channel with $\kappa_{c}=0.125220$, extracted with the variational analysis method from an operator basis excluding (left) and including (right) scattering in mesons with strange valence quarks. The results are obtained from 125 configurations of the U101 ensemble.

light quark mass and an unphysically light strange quark mass, therefore the resulting analysis of the scattering amplitude will necessarily have to take into account the additional scattering channels given by strange mesons.

\section{Conclusions}

We reported on the non-perturbative lattice determination of the properties of the resonances in the charmonium spectrum. We studied the impact of the tuning of the charm quark mass on the lattice energy levels, finding evidences that modifying $\kappa_{c}$ might be crucial to move the position of the $\bar{D} D$ threshold below the second exited states in the vector channel. The preliminary results of the energy spectrum show that our target statistics are sufficient to reliably estimate the scattering states relevant for extracting the scattering amplitudes. The calculations of the contractions on the remaining configurations as well as the analysis itself is ongoing. The plan for the near future is to provide an analysis of scalar and vector charmonium resonances on the two ensembles U101 and H105, including scattering in moving frames.

\section{Acknowledgements}

Our code that implements the distillation method is written within the framework of the Chroma software package [19]. The inversion of the Dirac operator is performed used the multigrid solver of Ref. [20-23]. The simulations were performed on the Regensburg iDataCool cluster, and the SFB/TRR 55 QPACE 2 [24] and QPACE 3 machines. We thank our colleagues in CLS for the joint effort in the generation of the gauge field ensembles which form a basis for the here described computation. The Regensburg group was supported by the Deutsche Forschungs- gemeinschaft Grant No. SFB/TRR 55. M. P. acknowledges support from EU under grant no. MSCA-IF-EF-ST-744659 (XQCDBaryons). 


\section{References}

[1] M. Lüscher, Commun. Math. Phys. 105, 153 (1986); M. Lüscher, Nucl. Phys. B354, 531 (1991); M. Lüscher, Nucl. Phys. B364, 237 (1991).

[2] BesIII Collaboration, Phys. Rev. Lett. 118 (2017), 092001.

[3] Belle Collaboration, Phys. Rev. Lett. 94(2005), 182002; Babar Collaboration, Phys. Rev. Lett. 101 (2008) 082001; Belle Collaboration, Phys. Rev. Lett. 104(2010) 092001

[4] J. Beringer et al. (Particle Data Group), Phys. Rev. D86, 010001 (2012) and 2013 partial update for the 2014 edition.

[5] J. P. Lees et al. [BaBar Collaboration], Phys. Rev. D 86, 072002 (2012) doi:10.1103/PhysRevD.86.072002].

[6] Feng-Kun Guo, Ulf-G.Meißner, "Where is the $\chi_{c 0}(2 P)$ ?", Phys. Rev. D 86 (2012), 091501; Stephen Lars Olsen, "Is the X(3915) the $\chi_{c 0}(2 P)$ ?", Phys. Rev. D 91(2015), 057501

[7] L. Liu, G. Moir, M. Peardon, S. M. Ryan, C. E. Thomas, P. Vilaseca, J. J. Dudek, R. G. Edwards, B. Joó, D. G. Richards, "Excited and exotic charmonium spectroscopy from lattice QCD", JHEP 07 (2012) 126.

[8] Zhi-Gang Wang, "Scalar tetraquark state candidates: X(3915), X(4500) and X(4700)", Eur. Phys. J. C77 (2017) 78.

[9] Xin Li and M. B. Voloshin, "X(3915) as a $D_{s} \bar{D}_{s}$ bound state”, Phys. Rev. D 91, 114014 (2015).

[10] Z. Y. Zhou, Z. Xiao and H. Q. Zhou, Phys. Rev. Lett. 115, no. 2, 022001 (2015).

[11] C. Patrignani et al. "Review of Particle Physics". In: Chin. Phys. C40.10 (2016).

[12] K. Chilikin et al. [Belle Collaboration], Phys. Rev. D 95, 112003 (2017).

[13] C. B. Lang, L. Leskovec, D. Mohler and S. Prelovsek, "Vector and scalar charmonium resonances with lattice QCD,” JHEP 1509 (2015) 089.

[14] M. Bruno et al., "Simulation of QCD with $\mathrm{N}_{f}=2+1$ flavors of non-perturbatively improved Wilson fermions," JHEP 1502 (2015) 043.

[15] G. S. Bali et al. [RQCD Collaboration], "Lattice simulations with $N_{f}=2+1$ improved Wilson fermions at a fixed strange quark mass," Phys. Rev. D 94 (2016) no.7, 074501.

[16] M. Peardon et al. [Hadron Spectrum Collaboration], "A Novel quark-field creation operator construction for hadronic physics in lattice QCD,” Phys. Rev. D 80 (2009) 054506.

[17] S. Prelovsek, G. Bali, S. Collins, D. Mohler, M. Padmanath, S. Piemonte, and S. Weishaeupl, "Charmonia in moving frames", in Proceedings, 35th International Symposium on Lattice Field Theory (Lattice2017): Granada, Spain, to appear in EPJ Web Conf.

[18] C. Morningstar, J. Bulava, J. Foley, K. J. Juge, D. Lenkner, M. Peardon and C. H. Wong, Phys. Rev. D 83, 114505 (2011).

[19] R.G. Edwards, B. Joo (SciDAC, LHPC, UKQCD), "The Chroma Software System for Lattice QCD”, Nucl. Phys. Proc. Suppl. 140, 832 (2005).

[20] S. Heybrock, B. Joó, D.D. Kalamkar, M. Smelyanskiy, K. Vaidyanathan, T. Wettig, P. Dubey, "Lattice QCD with Domain Decomposition on Intel Xeon Phi Co-Processors", SC '14: Proceedings of the International Conference for High Performance Computing, Networking, Storage and Analysis.

[21] S. Heybrock, M. Rottmann, P. Georg, T. Wettig, "Adaptive algebraic multigrid on SIMD architectures", PoS LATTICE2015, 036 (2016).

[22] D. Richtmann, S. Heybrock, T. Wettig, "Multiple right-hand-side setup for the DD- $\alpha$ AMG ", PoS LATTICE2015, 035 (2016). 
[23] P. Georg, D. Richtmann, T. Wettig, "pMR: A high-performance communication library", PoS LATTICE2016, 361 (2017).

[24] P. Arts et al., "QPACE 2 and Domain Decomposition on the Intel Xeon Phi”, PoS LATTICE2014, 021 (2015). 